\title{
A Novel Calibration Method in the Presence of Space Charge in Dielectric Materials Using the Pulsed Electroacoustic Technique
}

\author{
G. Chen ${ }^{1 *}$, Y. L. Chong ${ }^{2}$ and M. Fu ${ }^{3}$ \\ ${ }^{1}$ School of Electronics and Computer Science \\ University of Southampton UK \\ ${ }^{2}$ Data Storage Institute \\ Singapore \\ ${ }^{3}$ Department of Engineering \\ University of Leicester UK \\ *Email: gc@ecs.soton.ac.uk
}

\begin{abstract}
Calibration is an important part of procedures to accurate measurements of space charge in solid dielectrics. The pulsed electroacoustic (PEA) technique is one of the mostly used techniques due to its simplicity in construction, low cost and easy to implement for both plaque and cable samples. A novel method has been proposed which enables the PEA technique to be used in the presence of space charge. It is based on two measurements. Firstly, the sample with the presence of charge is measured without any applied voltage. The second measurement is carried out with a small dc applied voltage. The applied voltage should be small enough so there is no disturbance on the existing charge in the sample. The difference of the two measurements can be used for calibration. The additional advantage of the proposed method avoids the influence of the pulse voltage on calibration. A $200 \mu \mathrm{m}$ LDPE sample was stressed at $8 \mathrm{kV}$ for 60 minutes to produce a significant amount of charge in the sample. The proposed method has been used to calculate the residual charge and the result has been compared with the conventional method.
\end{abstract}

\section{INTRODUCTION}

Solid polymeric materials, such as polyethylene (PE), cross-linked polyethylene (XLPE) and polypropylene (PP), are now being widely used in high voltage power apparatus as insulation. The advantages of such materials are their high dielectric strength and electrical resistivity combined with good physical properties, such as resistance to cracking and moisture penetration. However, under certain operating conditions, their good electrical insulation properties may become degraded. For example, trapped or low mobility electrically charged species within the bulk can give rise to space charge, resulting in localised electric stress enhancement. This can cause further concentration of charge and lead to premature failure of the material [1]
Recent developments in space charge measurement technique have enabled space charge in solid materials determined non-destructively. The PEA technique is more common than the other techniques due to its simplicity in structure, low cost and easy to implement for both plaque and cable samples. To quantify space charge in the material, calibration on measured signal is required. However, the calibration of samples in the presence of space charge has never been addressed.

In the present paper a normal procedure used for charge density calibration using the PEA technique is briefly described. Our main focus is to deal with one of the practical situations where space charge exists in the material prior to any measurements. A new method based on two step measurements is proposed. An experiment is devised to validate the proposed method.

\section{PEA SYSTEM MODEL AND ITS FREQUENCY REPRESENTATION}

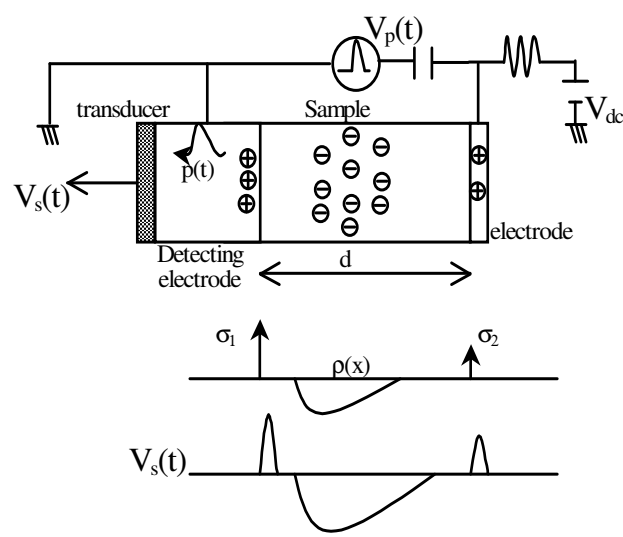

Fig.1 Schematic diagram of PEA system

The details of a PEA system can be found in literatures [2] and a schematic diagram is shown in Fig.1. In summary, acoustic waves are produced at charge layers at both electrodes and internal charge when an electrical pulse is applied to a sample. The acoustic signals are detected by a piezo-electric transducer. The electric 
signal obtained in time domain represents the charge distribution [2]:

$$
V_{s}(t)=K\left[\sigma_{1}+\sigma_{2}+v_{s a} \Delta T \rho\left(x=v_{s a} t\right)\right] e_{p}
$$

where $\sigma_{1}$ and $\sigma_{2}$ are the surface charges at the electrodes, $v_{\mathrm{sa}}$ the sound velocity through the material, $\Delta \mathrm{T}$ the width of the pulse, $\rho$ the bulk charge and $e_{p}$ the amplitude of the pulse voltage.

Quantitative charge analysis needs the system to be calibrated, i.e. $\mathrm{K}$ needs to be determined. $\mathrm{K}$ is determined by many factors such as acoustic properties of materials encountered by acoustic waves, and electrical parameters of the measuring system. In theory, it is extremely difficult to obtain an analytical solution due to complexity of the system. Therefore, it is often determined experimentally. For a planar sample if there is no bulk charge in the material the third term in equation (1) is zero, therefore, the output signal at the electrodes can be written as

$$
\begin{aligned}
& V_{1}=K \sigma_{1} e_{p} \\
& V_{2}=K \sigma_{2} e_{p}
\end{aligned}
$$

Both equations can be used to determine the constant $\mathrm{K}$ if the acoustic attenuation and dispersion are negligible. However, in practice equation (2), the signal from the electrode closer to the transducer, is often used as acoustic signal experiences less losses.

Recently a model has been proposed [3] where the PEA system in most of cases can be treated as a linear time-invariant (LTI) system. As this is important from calibration point of view it is worth of reviewing the model briefly. It is well known [4] that the impulse response $\mathrm{h}(\mathrm{t})$ of the LTI system is simply the output when the input is the unit impulse sequence $\delta(t)$ and the impulse response is a complete characterisation of any LTI system. Convolution is the general formula that allows us to compute the output $\mathrm{y}(\mathrm{t})$ from the input $\mathrm{x}(\mathrm{t})$ for any LTI system. This can be illustrated in Fig.2.

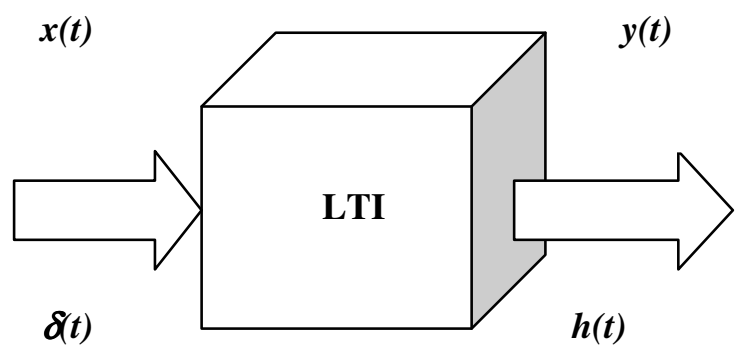

Fig.2 Linear time-invariant system with impulse input.
In mathematical terms it can be represented by

$$
y(t)=\int x(\tau) h(t-\tau) d \tau=x(t) * h(t)
$$

where * represents an operation of the convolution.

In practice, the signal detected by the sensor is small and needs to be amplified. The sensor and the input impedance of the amplifier constitute a typical high-pass filter. This filter is another LTI system. Therefore the PEA system including the detecting circuit can be viewed as two LTI systems connected in cascade. One of the important properties of LTI systems is that the cascaded LTI systems behave as a new LTI system [4]. In a cascaded connection of two LTI systems, the output of the first system is the input of the second system, and the overall output of the cascaded system is the output of the second system. Assuming that the impulse response of the first LTI system is $h_{1}(t)$ and the second LTI system $h_{2}(t)$, the overall impulse response of the cascaded system will be $\mathrm{h}_{1}(\mathrm{t}) * \mathrm{~h}_{2}(\mathrm{t})$. Therefore equation (4) is still valid even including the response of the detecting circuit. It has been found [5] that an easy computation can be achieved by converting the signal from time domain to frequency domain. The equation (4) in frequency domain can be represented as

$$
Y(f)=X(f) H(f)
$$

where $\mathrm{H}(\mathrm{f})$ is the impulse response of the system including the sensor and amplifier in frequency domain.

Experimentally, it is impossible to produce a unit impulse $\delta(\mathrm{t})$ which means $\mathrm{H}(\mathrm{f})$ cannot be found. However, from equation (5), the characteristics of the system can be obtained by injecting a known X(f) and measuring $\mathrm{Y}(\mathrm{f})$. In order to obtain a broad spectrum, a narrow electric pulse is often applied to the system. Once H(f) is obtained, its effect on the output signal can be removed (known as deconvolution).

\section{CALIBRATION}

Calibration is important if quantitative charge distributions are required. This becomes growing important as the understanding of the effect of space charge on electrical ageing is improving. There are several methods that can be used depending on the actual sample in question. In some cases it is not an easy task to perform a calibration. In the following sections calibration procedures have been considered according to the sample status.

\section{Sample without Space Charge}

This is the situation for most of the measurements. The conventional method is to apply a low de voltage prior 
to any other measurements. A typical example is shown in Fig. 3 where $2 \mathrm{kV}$ is applied to $200 \mu \mathrm{m}$ LDPE sample and measurement was made in less than 5 seconds. At such a low electric field and short time there should not be any charge generated in the material. This means the peak between two electrodes is response of the system. By applying deconvolution and calibration mentioned in the previous section the response of the system can be eliminated and the signal scaled to charge density as shown in Fig. 4.

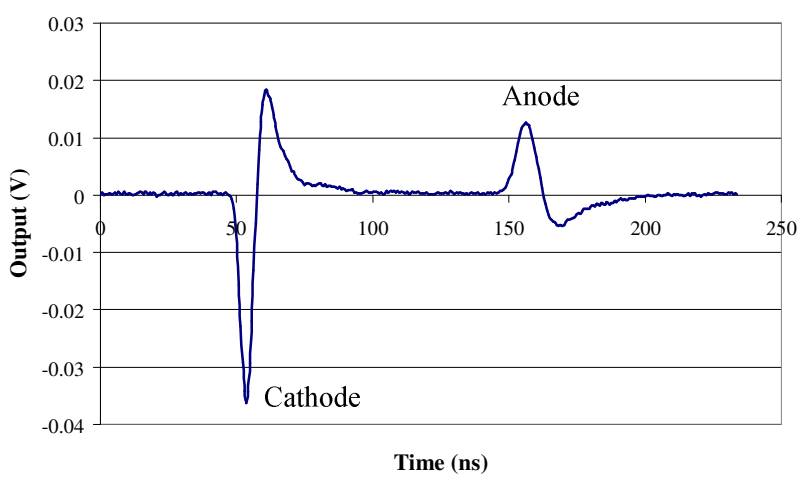

Fig.3 PEA output of $200 \mu \mathrm{m}$ LDPE at $2 \mathrm{kV}$.

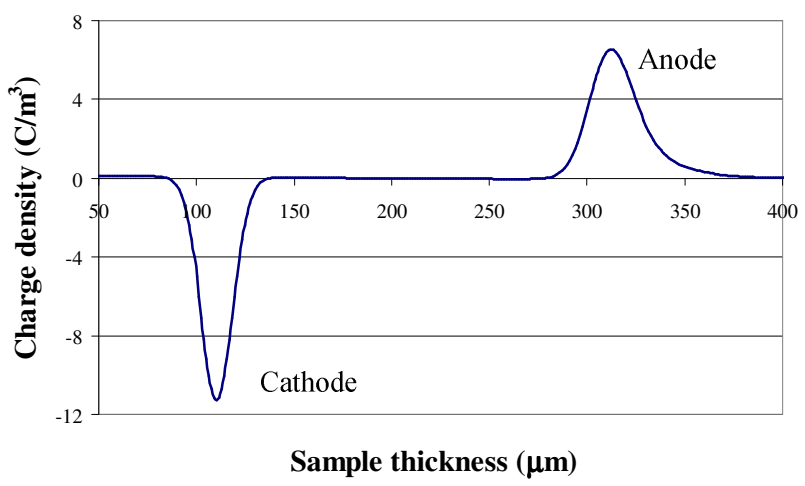

Fig. 4 Calibrated charge distribution in LDPE.

As expected, there is no bulk charge at this low electric field. Further measurements have to be processed in a similar fashion so the quantitative space charge information can be revealed.

\section{Sample with Trapped Charge}

In some cases where charge is trapped either in the bulk or on the surfaces it is still possible to calibrate so a quantitative charge distribution can be obtained. There are two methods available. The first solution to this problem is to use the same batch of samples without charge for calibration purpose. This method has been used in literature [6]. In theory this method sounds very reasonable, however, in practice as there are several interfaces involved in the PEA system which have a significant influence on the propagation of acoustic waves. Consequently there are considerable variations in the output signal from sample to sample due to slight differences in sample assembling. It is, therefore, always desirable that the calibration and subsequent measurements are carried out on the sample without involving sample loading and unloading.

The second method proposed below can be applied to the case where deeply trapped charge is involved. Charges can be roughly classified as fast and slow charge according to mobility. Charges trapped in the insulating material are often stable i.e. slow charge. In the situation like this the calibration has to take two steps involving measurement with 'volts on' and 'volts off' ( $\mathrm{dc}$ voltage). The subtraction between the two measurements gives the output signal which excludes the contribution from the trapped charge, in other words, it only represents the effect of dc voltage, therefore the calibration can be done in a similar way as those described earlier.

Assuming that the signal on the electrode near to the transducer is $\mathrm{V}_{1 \text {-on }}$ and $\mathrm{V}_{1-\text { off }}$ respectively corresponding to volts on and off, according to equation (2) we have

$$
\begin{gathered}
V_{1-o n}=K e_{p}\left(\sigma+\delta+\sigma_{1}\right) \\
V_{1-o f f}=K e_{p}(\sigma+\delta)
\end{gathered}
$$

where $\sigma$ represents the induced charge on the electrode due to trapped charge in the sample, $\delta$ the charge due to the pulse voltage and $\sigma_{1}$ the capacitive charge due to the applied dc voltage.

By performing (6)-(7), the effect of the trapped space charge as well as the pulse voltage on the signals can be removed and this gives

$$
V_{1-o n}-V_{1-o f f}=K e_{p} \sigma_{1}
$$

Except from K, the rest of variables in the above equation can be either measured or known from the applied voltage, therefore $\mathrm{K}$ can be calculated. Once K is known, the space charge in the bulk of the sample can be estimated based on equation (7).

\section{EXPERIMENTAL RESULTS}

To validate the above calibration procedure, an experiment was devised to produce a sample with trapped charges. In this experiment an unaged LDPE of approximately $200 \mu \mathrm{m}$ thick was used. A conventional calibration was performed at $1 \mathrm{kV}$ dc voltage before 8 $\mathrm{kV} \mathrm{dc}$ voltage was applied to the sample for 60 minutes. Measurements were carried out at various times during stressing and after the removal of the applied voltage.

The results during stressing indicate homocharges formation in the vicinity of both electrodes. The origin of these homocharges is believed to be charge injected 
and subsequently trapped near the electrodes. It was observed that the amount of charge build up increases with ageing duration. As our main purpose here is to produce a sample which has trapped charges inside, the ageing test was terminated at 60 minutes. The charge decay in the aged sample was monitored and it has been noticed that there is a significant reduction of the amount of homocharges trapped in the material initially. These charges are believed to be trapped in shallow traps during stressing period. A steady reduction in the rate at which space charge decays can be observed until it becomes reasonably stable after 15 minutes.

Since the space charge profile after 15 minutes of charge decay is reasonably stable, it satisfies the condition of that the charge is trapped reasonably deep; as such can be considered as a sample with trapped charge. Therefore, the method proposed in section 3.2 can be applied.

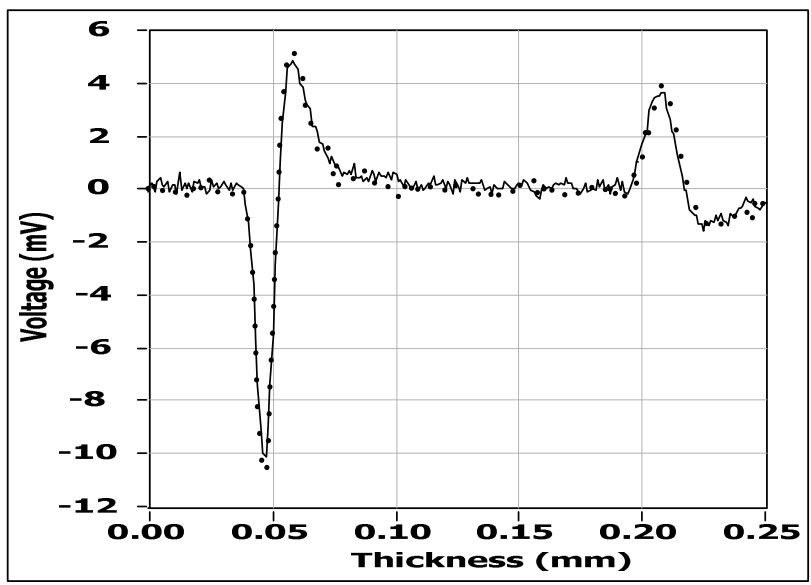

Fig. 5 Comparison of calibration signals between the signal obtained during the initial step voltage (solid line) and that obtained in the presence of trapped charge (dotted line).

It should be stressed that in order to obtain a suitable volts on signal, the applied voltage must be high enough so as to yield a signal of acceptable signal-to-noise ratio. However, it is also important that the magnitude of the applied voltage should be low such that the charge originally accumulated is not significantly affected. Furthermore, applying too high a voltage may also likely to cause fast charge accumulation in the sample. A suitable voltage for the present sample was found to be approximately $1 \mathrm{kV}$.

A comparison of calibration signals between the signal obtained using the conventional method (solid line) and the signal obtained using the procedure described earlier (dotted line) is shown in Fig. 5. For the latter, a measurement was performed on the sample after it was decayed for 15 minutes, then a $1 \mathrm{kV}$ dc voltage was applied to the sample. It can be seen that there is little difference between the two signals, even to the extent that they almost overlapped each other when put together. This implies that there is little fast charge accumulation in the sample when a $1 \mathrm{kV}$ dc voltage was applied. More importantly it validates the procedure described earlier.

Using the calibration signal obtained we have processed the data obtained from the decay test. Not surprisingly, the result is almost identical to the one processed from the initial calibration signal as shown in Fig. 6.

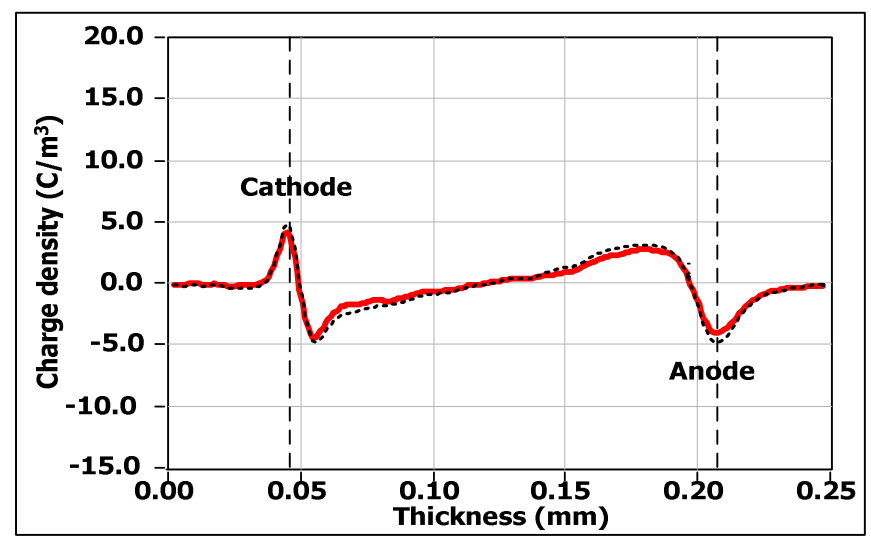

Fig. 6 Trapped charge in the sample calculated using the method proposed (solid line) and conventional method (dotted line).

\section{CONCLUSION}

The proposed new calibration method for charge distribution using the PEA technique in the sample with trapped charge is very effective and accurate.

\section{REFERENCES}

[1] Z. Liu, R. Liu, H. Wang and W. Liu, "Space charge and initiation of electrical trees", IEEE Trans. Elec. Insul., Vol. 24, pp. 83-89, 1989.

[2] Y. Li, M. Yasuda and T Takada, "Pulsed electroacoustic method for measurement of charge accumulation in solid dielectrics", IEEE Trans. Dielectrics and Electrical Insulation, Vol.1, pp. 188 195, 1994.

[3] Y. Zhu, D. Tu and T. Takada, "Mathematical analysis and interpretation of Pulsed Electro-acoustic System", 6 ${ }^{\text {th }}$ ICPADM, Xian, China, pp. 63 - 66, 2000.

[4] J. C. Proakis and D. G. Manolakis, Digital Signal Processing - Principles, Algorithms, and Applications, $3^{\text {rd }}$ edition, Prentice-Hall, 1996.

[5] A. Vazquez, G. Chen, A. E. Davies and R. Bosch, "Space charge measurement using PEA technique and signal recovery", J. European Ceramic Society, Vol.19, pp.1219-1222, 1999.

[6] T. Maeno, "Calibration of the pulsed electroacoustic method for measuring space charge density", Trans. IEE Japan, Vol. 119-A, pp. 1114 - 1119, 1999. 